\title{
Determination of Salmonella pullorum with Nanoparticles Immune Based Lateral Flow Strip Assay
}

\author{
Bin Wu ${ }^{1 *}$, Xiuyun Zhang1, Wei Pan², Lin Zhang2, Fan Zhang² \\ ${ }^{1}$ Liaoning Entry-Exit Inspection and Quarantine Bureau, Dalian, China \\ ${ }^{2}$ Chinese Academy of Inspection and Quarantine, Beijing, China \\ Email: wubin69@163.com
}

Received 9 April 2015; accepted 18 May 2015; published 25 May 2015

Copyright (C) 2015 by authors and Scientific Research Publishing Inc.

This work is licensed under the Creative Commons Attribution International License (CC BY).

http://creativecommons.org/licenses/by/4.0/

(c) ()

\section{Abstract}

The isolation and culture of conventional detection method of salmonella can not meet the testing requirements of quick and easy detection at the grassroots level. In this study, we prepare the fluorescent nanoparticles as a marker, covalently conjugate with monoclonal antibodies of Salmonella pullorum. The whole Salmonella pullorum antigen and goat anti-mouse antibody sprayed on the nitrocellulose membranes are used as test line and control line. The fluorescence nanoparticles immune based lateral flow strips are made according to the principle of antigen-antibody immune response. The test strips may interpret results within $30 \mathrm{~min}$. The results of the salmonella A, S. agona, S. chester and S. arechavaleta are positive, including, S. agona for weakly positive. After analysis, it is found that in addition to the salmonella of group $A$, the other positive salmonella are in group B. But it is negative of S. derby, S. rissen, and other 6 kinds of salmonella, with good specificity. The fluorescence nanoparticles immune based lateral flow strips are a little of sample can be detected fast, easily, inexpensive, easy to universal without professional technical personnel detection method. It provides a new detection method for the detections of Salmonella pullorum.

\section{Keywords}

Monoclonal Antibody, Nanoparticles, Fluorescence Nanoparticles Immune Based Lateral Flow Strips, Salmonella pullorum

\section{Introduction}

Fowl Pullorosis (Pullorum Disease) also known as pullorum or pullorum disease, is an infectious disease of

\footnotetext{
"Corresponding author.
}

How to cite this paper: Wu, B., Zhang, X.Y., Pan, W., Zhang, L. and Zhang, F. (2015) Determination of Salmonella pullorum with Nanoparticles Immune Based Lateral Flow Strip Assay. Advances in Microbiology, 5, 364-369. 
poultry disease caused by Salmonella pullorum. In addition to the disease level of broadcasting, mainly through the egg transfer, is a vicious spiral type throughout the period of disease of chicken. The disease can cause the fertilization rate and hatching rate and laying rate decreased, causing significant harm to the poultry industry. The prevalence of chicks to Salmonella pullorum for features, often manifested as acute symptoms of septicemia. OIE will be classified as class B disease, China's Ministry of agriculture listed as the two kind of disease [1]. Salmonella enterobacteriaceae genus has at least 2300 serotype. Based on the difference of antigen by bacteria, salmonella can be divided into A, B, C, D, E, F seven groups [2]. One of the most common pathogenic is group B, Pigs cholera in group C and pullorum and Salmonella enteritidis in group D [3]. Conventional detection methods of Salmonella pullorum by the disadvantages of long cycle and complicated operation has been far can not meet the requirements of current detection, not suitable for field detection method of port, so there is an urgent need for a rapid and accurate detection of Salmonella pullorum. Nano fluorescent strip is currently on a fast screening method for model [4], can be used for rapid diagnosis of some infectious diseases, for suspected infection in chicken flocks in the early disease receive timely treatment [5].

\section{Materials and Methods}

\subsection{Test Materials}

(3-Aminopropyl) trimethoxylsilane (APTMS), tetraethyl orthosilicate (TEOS), TritonX-1004, 4-bis,1,1,2,2,3,3heptafluoro-4,6-hexanedion-6-yl) chlorosulfo-o-terphenyl (BHHCT) and Sodium Cyanoborohydride are bought in Sigma. The nitrocellulose membrane is bought in Millipore. S. pullorum monoclonal antibody is bought in Biodesign. S. pullorum and other reference strains of inactivated strain are from the city center for disease control and prevention. The other reagents were domestic analytical reagent.

\subsection{Test Instrument}

JEM 2100 HC electron microscope; Perkin Elmer-LS-55 fluorescence spectrophotometer; Ultraviolet imager (UVP company, USA); Canon Powershot A610 digital camera.

\subsection{Test Method}

\subsubsection{The Preparation of Fluorescent Nanoparticles}

The preparation of fluorescent nanoparticles [6] [7] process is shown in Figure 1.

\subsubsection{The Antibody Markers}

The antibody markers [8] flow diagram, as shown in Figure 2.

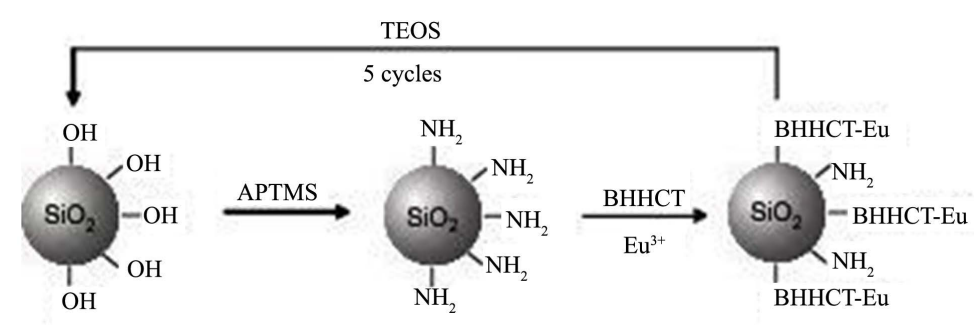

Figure 1. The flow diagram of the preparation of fluorescent nanoparticles.

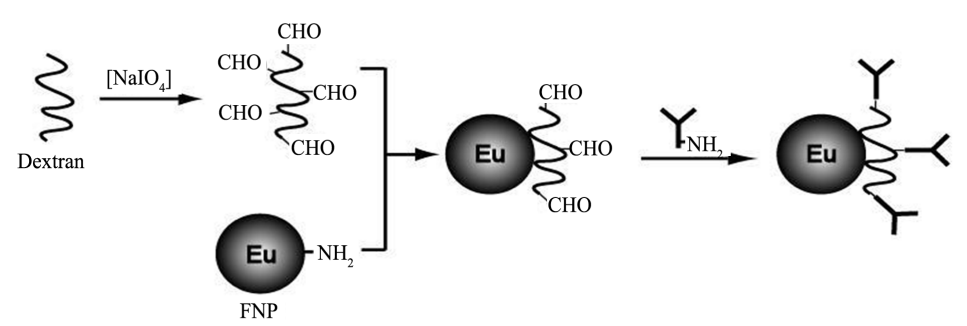

Figure 2. The antibodies labeled flow diagram. 


\subsubsection{The Immune Based Lateral Flow Strips [9]}

1) The labeled antibody into solid phase

The good marker particles of preparation with $1 \%$ casein diluent makes up of $10 \mathrm{mM}$ Tris 7.8 buffer according to 1:1000 diluted; Then the glass fiber as markers mat immersing among them, will be subject to soak, and then freeze-dried for later use.

2) Package capture antibody

The membrane dispenser system will be for $2 \mathrm{mg} / \mathrm{mL}$ antibodies into linear or strip on the nitrocellulose membrane, the package volume is $0.7 \mu \mathrm{L} / \mathrm{cm}$ as detection line; $0.5 \mathrm{~cm}$ from the detection line on the membrane is packaged by $2 \mathrm{mg} / \mathrm{mL}$ sheep against mouse IgG to draw a quality control line, dry reserve at $37^{\circ} \mathrm{C}$ for $2 \mathrm{~h}$.

3) Nanoparticles marker combination mat processing and related material of choice.

4) The structure diagram of the strip is shown in Figure 3.

5) Samples detection

Add $60 \mu \mathrm{L}$ sample which to be tested on the strip mat. After 20 min, detecting the strip under UV lamp observations or measuring degree by fluorescence detector. If the sample target content is low, it will need to extend the time. The positive result presents two red belts, and the negative result shows only a red belt in 30 min. In this experiment, we use Canon Powershot A610 digital camera for auxiliary observation and record the results.

\section{Test Results}

\subsection{The Preparation and Characterization of Fluorescent Nanoparticles}

From the Figure 4, we can see the morphology of the blank silica nanoparticles made by reverse micelle method is in good condition. The size is between $(60 \pm 5 \mathrm{~nm})$, The surface of the particle is smooth.

\subsection{Sensitivity Test}

The strain used in this test, are all inactivated strain after boiled. We used the real-time fluorescence PCR to detect $S$. pullorum, and then it will be diluted to different concentrations to examine the sensitivity of the detection system. The result is shown in Figure 5, the detection sensitivity of the strips can reach $5 \times 10^{3}$ copies $/ \mathrm{mL}$. The two highest detection concentrations showed decline fluorescence phenomenon in Figure 5.

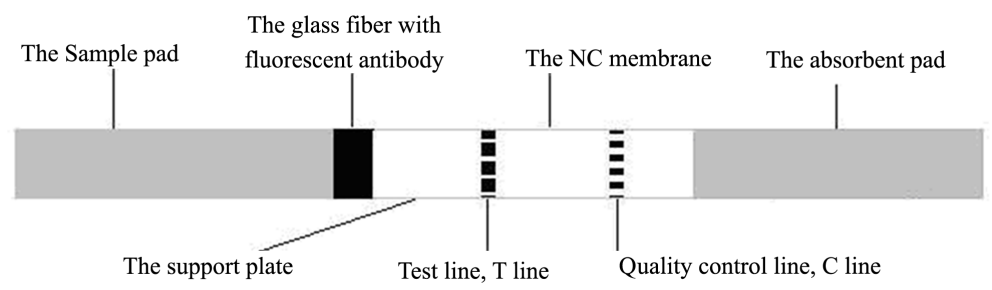

Figure 3. The structure diagram of the strip.

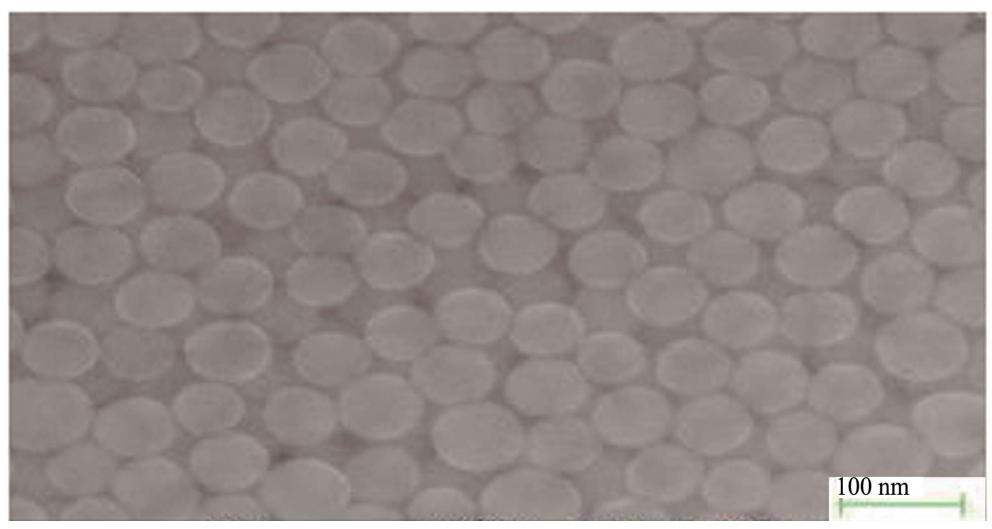

Figure 4. The SEM photo of silicon nanoparticles. 


\subsection{Specific Test}

First, we use the 16 collected strains of not salmonella to investigate the specificity of the detection system. They are: Escherichia coli, E. coli 8099, EPEC (pathogenic E. coli), EPEC O125:K70, EPEC O128:K67, EPEC O44:K74, EIEC (enteroinvasive E. coli) O164, EIEC O28:K73, E. coli O157:H7, Sh. flexneri 2a, Vibrio parahaemolyticus, VP, Vibrio cholerae O1, Vibrio cholerae O139, Staphylococcus aureus, Listeria monocytogenes and Yersinia enterocolitica. As shown in Figure 6, the detection system of the 16 strains of not salmonella without nonspecific reaction.

Then, we make use of the collected 12 strains of salmonella and different species to inspect the interspecific specificity of detection system. They include: S. pullorum, the Salmonella D, S. agona, the Salmonella A, the Salmonella E, S. eastborne, S. rissen, S. weherreden, S. chester, S. anatum, S. chailey and S. panama. The result is shown in Figure 7, besides the result of $S$. pullorum is positive, the results of the salmonella $D$, S. eastborne and S. panama are positive, too. After analysis, it is found that in addition to the salmonella of group D, the other positive salmonella are in group D. Salmonella group is classified according to the difference of LPS, and chooses the monoclonal antibody of S. pullorum resistance loci for LPS, so the D group with S. eastborne and $S$. panama are also cross reaction.

\subsection{Selective Test}

This experiment mainly inspects to detect the $S$. pullorum from other strains of higher interference environment infected in chickens artificially. The background of E. coli O157:H7, keep the total concentration of $5 \times 10^{8} / \mathrm{mL}$ unchanged in this experiment. The proportion of the content of the S. pullorum is changed from $50 \%$ to $0.001 \%$, investigating the sensitivity. From the Figure 8 you can see, the detection sensitivity of the system can still achieve $5 \times 10^{3}$ copies $/ \mathrm{mL}$. Below diagram is about two groups of parallel test.

\section{Test Conclusions}

This paper established "fluorescent nanoparticles" detection technology platform based on S. pullorum detec-

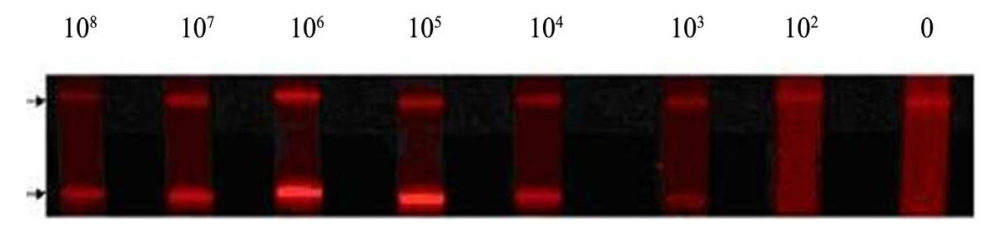

Figure 5. The sensitivity test.
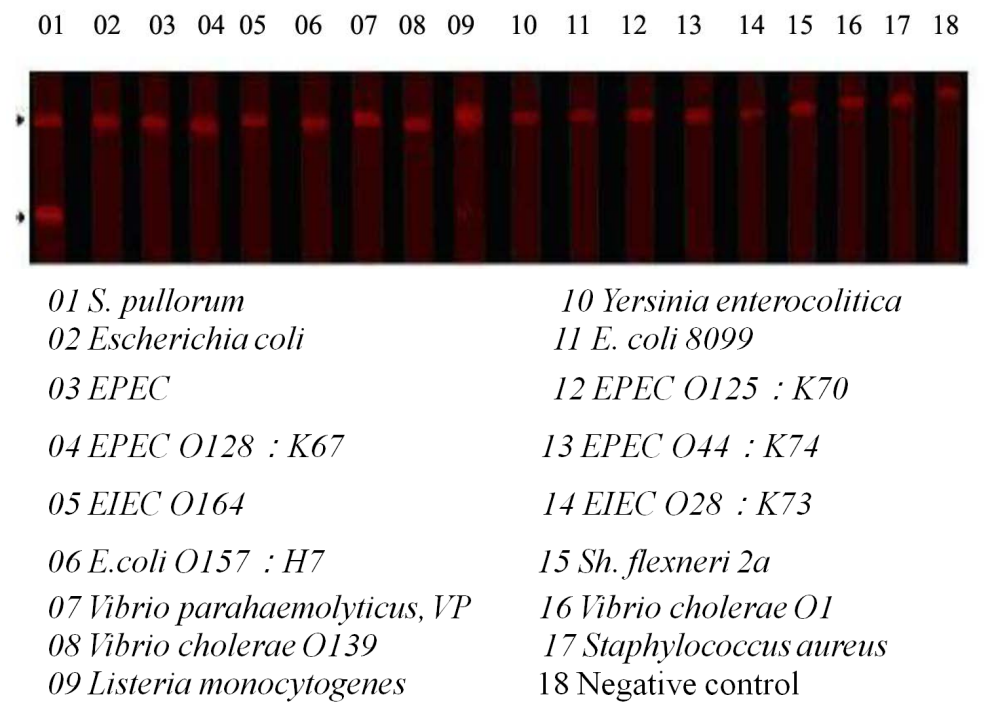

Figure 6. The specificity test. 


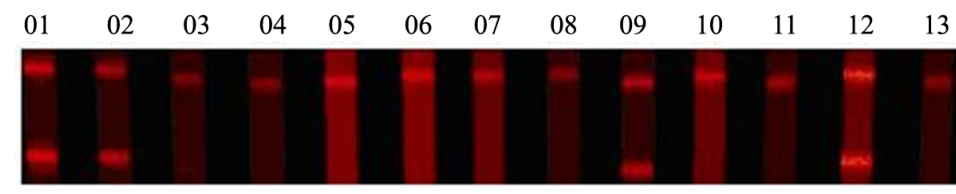

$\begin{array}{ll}01 \text { S. pullorum } & 07 \text { the Salmonella A } \\ 02 \text { the Salmonella D } & 08 \text { S.agona } \\ 03 \text { the Salmonella E } & 09 \text { S.eastbourne } \\ 04 \text { S.rissen } & 10 \text { S. Weherreden } \\ 05 \text { S.chester } & 11 \text { S.anatum } \\ 06 \text { S.chailey } & 12 \text { S.panama } \\ & 13 \text { Negative control }\end{array}$

Figure 7. The salmonella specificity test between species.

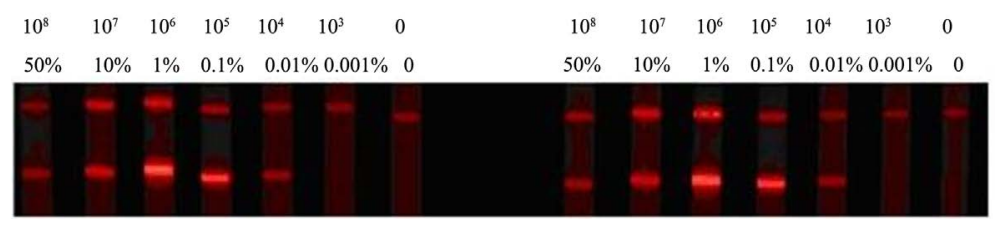

Figure 8. The selective test of detection system.

tion object. Its related principle and technology can be applied to other harmful microbial detection. And with the further development of research in the future, it can be achieved through synthesis of multicolor fluorescence nanoparticles on a variety of harmful microbial detection at the same time, in order to achieve rapid, sensitive and high throughput detection. In this paper, it is made the nano gold test strips for S. pullorum. And the experiments of detection sensitivity, repeatability and specificity and stability of the strips obtained good results.

This paper established a technology platform versatility. Comparing with the existing products, the nanoparticles immune based lateral flow strips have obvious competitive. The product can be widely used in healthy and epidemic prevention, food, agriculture, animal husbandry, import and export inspection and quarantine, and other fields.

\section{Fund Project}

The state administration of quality supervision, inspection and quarantine of scientific research projects (2012IK013, 201410059).

\section{References}

[1] Jeon, S., Eo, S.K., Kim, Y., Yoo, D.J. and Kang, S.H. (2007) Capillary and Microchip Gel Electrophoresis for Simultaneous Detection of Salmonella pullorum and Salmonella gallinarum by rfb S Allele-Specific PCR. 73, 415-419.

[2] Wang, J. (2007) Nanoparticle. Based Electrochemical Bioassays of Proteins. Electroanalysis, 19, 769-776. http://dx.doi.org/10.1002/elan.200603789

[3] Wu, H., Liu, G., Wang, J., et al. (2007) Quantum-Dotsbased Electrochemical Immunoassay of Interleukin. Electrochemistry Communications, 9, 1573-1577. http://dx.doi.org/10.1016/j.elecom.2007.02.024

[4] Ding, S.Y., Jones, M., Tucker, M.P., et al. (2003) Quantdot Molecules Assembled with Genetically Engineered Proteins. Nano Letters, 3, 1581-1585. http://dx.doi.org/10.1021/nl034578t

[5] Zajac, A., Song, D.S., Qian, W., et al. (2007) Protein Microarrays and Quan Tum Dot Probes for Early Can Detection. Colloids and Surfaces B: Biointerfaces, 58, 309-314. http://dx.doi.org/10.1016/j.colsurfb.2007.02.019

[6] Chen, J.X., Ye, Z.Q., Wang, G.L. and Yuan, J.L. (2009) A Hydrogen Peroxide Fluorescence Application of Rare Earth Nanometer Silicon Dioxide. Analytical Chemistry, 10, 23-26. 
[7] Lucas, L.J., Chesler, J.N. and Jeonyeol, Y. (2007) Labonachip Immunoassay for Multiple Antibodies Using Micmsphere Light Scattering and Quantum Dot Mission. Biosensors and Bioelectronics, 23, 675-681. http://dx.doi.org/10.1016/j.bios.2007.08.004

[8] Duan, H.-L., Shen, Z.-Q., Wang, X.-W., Chao, F.-H. and Li, J.-W. (2005) Preparation of Immunomagnetic Iron-Dextran Nanopartides and Application in Rapid Isolation of E. coli O157:H7 from Foods. World Journal of Gastroenterology, 24, 3660-3664. http://dx.doi.org/10.3748/wig.v11.i24.3660

[9] Preechakasedkit, P., Pinwattana, K., Dungchai, W., Siangproh, W., Chaicumpa, W., Tongtawe, P. and Chailapakul, O. (2011) Development of a One-Step Immunochromatographic Strip Test Using Gold Nanoparticles for the Rapid Detection of Salmonella typhi in Human Serum. Biosensors and Bioelectronics, 31, 562-566. http://dx.doi.org/10.1016/j.bios.2011.10.031 